\title{
Hydrogen-assisted fatigue crack propagation in a pure BCC iron. Part II: Accelerated regime manifested by quasi-cleavage fracture at relatively high stress intensity range values
}

\author{
Domas Birenis ${ }^{1, *}$, Yuhei Ogawa ${ }^{2,3}$, Hisao Matsunaga ${ }^{4,5,6}$, Osamu Takakuwa ${ }^{5}$, Junichiro Yamabe ${ }^{5,6,7}$, Øystein Prytz ${ }^{1}$, and \\ Annett Thøgersen ${ }^{8}$ \\ ${ }^{1}$ Department of Physics, Centre for Materials Science and Nanotechnology, University of Oslo, Blindern, NO-0316 Oslo, Norway \\ ${ }^{2}$ Graduate School of Engineering, Kyushu University, 744 Motooka, Nishi-ku, Fukuoka 819-0395, Japan \\ ${ }^{3}$ Research Fellow of the Japan Society for the Promotion of Science, 744 Motooka, Nishi-ku, Fukuoka 819-0395, Japan \\ ${ }^{4}$ Department of Mechanical Engineering, Kyushu University, 744 Motooka, Nishi-ku, Fukuoka 819-0395, Japan \\ ${ }^{5}$ Research Center for Hydrogen Industrial Use and Storage (HYDROGENIUS), Kyushu University, 744 Motooka, Nishi-ku, Fukuoka \\ 819-0395, Japan \\ ${ }^{6}$ International Institute for Carbon-Neutral Energy Research (I2CNER), Kyushu University, 744 Motooka, Nishi-ku, Fukuoka 819- \\ 0395, Japan \\ ${ }^{7}$ AIST-Kyushu University Hydrogen Materials Laboratory (HydroMate), 744 Motooka, Nishi-ku, Fukuoka 819-0395, Japan \\ ${ }^{8}$ SINTEF Materials and Chemistry PO Box 124 - Blindern, NO-0314 Oslo, Norway
}

\begin{abstract}
Hydrogen effect on fatigue performance at relatively high values of stress intensity factor range, $\triangle K$, of pure BCC iron has been studied with a combination of various electron microscopy techniques. Hydrogen-assisted fatigue crack growth rate is manifested by a change of fracture features at the fracture surface from ductile transgranular in air to quasi-cleavage in hydrogen gas. Grain reference orientation deviation (GROD) analysis has shown a dramatic suppression of plastic deformation around the crack wake in samples fatigued in hydrogen. These results were verified by preparing site-specific specimens from different fracture features by using Focused lon Beam (FIB) technique and observing them with Transmission Electron Microscope (TEM). The FIB lamella taken from the sample fatigued in air was decorated with dislocation cell structure indicating high amount of plasticity, while the lamella taken from the quasi-cleavage surface of the sample fatigued in hydrogen revealed a distribution of dislocation tangles which corresponds to smaller plastic strain amplitude involved at the point of fracture. These results show that a combination of critical hydrogen concentration and critical stress during fatigue crack growth at high $\Delta K$ values triggers cleavage-like fracture due to reduction of cohesive force between matrix atoms.
\end{abstract}

\section{Introduction}

Hydrogen has the potential to become one of the major energy carriers, but to become a feasible fuel alternative it has to close the gap against the traditional energy sources in terms of cost. One fraction of the high total cost of hydrogen fuel comes from the structural materials used in hydrogen industry. Industry is actively looking for cheaper materials and low-alloy or carbon steels with body-centered cubic (BCC) lattice structure are among the best contenders. Unfortunately, the understanding of hydrogen effect on these types of steels is still lacking [1] and the performance under cyclic loading in hydrogen environment is one of the most important properties which needs to be studied in detail for a successful realisation of BCC steels in hydrogen energy industry.

For steels in corrosive environment a stable crack growth region described by the Paris law in fatigue crack growth (FCG) curve usually shows a dual stage behavior. At relatively low $\Delta K$ values (in this article we refer to it as Stage I) the fatigue crack growth rate (FCGR) seems almost unaffected by environment, while at higher $\Delta K$ values (Stage II) the FCGR transitions into accelerated regime where the crack grows at higher rate. Such behavior is generally interpreted as a superposition of sustained load stress-corrosion cracking and pure mechanical fatigue [2]. Similar trends are applicable for fatigue crack growth in steels exposed to hydrogen gas [3]. According to fracture surface examinations the hydrogen-assisted cracking in high-strength steels manifests as a brittle intergranular fracture $[4,5]$, while low or medium-strength steels subjected to hydrogenassisted fatigue crack growth (HAFCG) show transgranular quasi-cleavage (QC) fracture accompanied by brittle-like striations on the fracture surfaces $[6,7]$. These observations resulted in several proposed models for explanation of the QC fracture formation 
mechanism $[6,8,9]$, which are based on the most common theories for HE, such as hydrogen-enhanced decohesion (HEDE) [10,11], hydrogen-enhanced localized plasticity (HELP) [12,13], hydrogen-enhanced stabilization of lattice defects $[8,14]$, or the combination of them. However, these models still lack direct and critical supporting evidence, mostly due to the fact that most of the experiments have been performed on materials with complex microstructures and several alloying elements. These factors make the interpretation difficult and thus, the analysis of the hydrogen effect on the fracture processes is further complicated.

In this study, in order to minimize complexities arising from microstructure and material impurity we chose to study pure Fe, which is the simplest system representing BCC steels. FCG data was acquired for specimens fatigued in air and in hydrogen gas at different pressures and then fracture surfaces, fracture paths and dislocation structures around the crack wake were investigated by using scanning electron microscopy (SEM) and transmission electron microscopy (TEM). Even though pure $\mathrm{Fe}$ in air demonstrated fully ductile transgranular fracture throughout the measured $\Delta K$ range, failure of the specimen in hydrogen was attributed to two essentially different types of fracture: intergranular (IG) at relatively low $\Delta K$ values and quasi-cleavage (QC) at relatively high $\Delta K$ values. This difference in fracture surfaces marked a two stage FCGR behavior with a different level of HAFCG acceleration and this article deals only with high $\Delta K$ regime (Stage II), while in Part I of this series of articles the Stage I regime is analyzed in detail [15]. The Grain Reference Orientation Deviation (GROD) analysis has shown a significant plastic deformation reduction in the samples fatigued in hydrogen in comparison with the ones fatigued in air. In addition, these results were verified by using TEM observations of thin foils extracted directly from QC fracture features. Namely, the foil extracted from sample fatigued in air was decorated with a dislocation cell structure which is a typical feature for heavily plastically deformed iron. On the other hand, in case of hydrogen the foils possessed a distribution of individual dislocations and dislocation tangles indicating a low plastic strain deformation and crack was confirmed to propagate along $\{100\}$ type cleavage plane. The combination of these results implies that hydrogeninduced cleavage cracking, resulting in a significant reduction of plastic deformation, was the main contributor to the HAFCG in stage II regime.

\section{Experimental}

The material and experimental methods were the same as described in Part I of this article series. Additionally, $\Delta K$-constant tests were conducted at $\Delta K=17 \mathrm{MPa} \cdot \mathrm{m}^{1 / 2}$ in air, $0.7 \mathrm{MPa}$ and $90 \mathrm{MPa}$ hydrogen. Also, a misorientation angle-dispersed plastic deformation distribution (MADPDD) technique was used to quantify plastic deformation. This technique uses the raw data from the GROD maps to plot the number of pixels versus misorientation angle, resulting in a MADPDD curve.

\section{Results and discussion}

\subsection{Fatigue crack growth data}

The FCG curves obtained from $\Delta P$-constant tests in air, nitrogen and different pressures of hydrogen gas are illustrated in Fig. 1. The crack growth curve is linear in logarithmic scale for air and nitrogen throughout the tested $\Delta K$ range, but in the case of hydrogen gas, there is an obvious distinction between Stage I (relatively low $\Delta K$ regime) and Stage II (higher $\Delta K$ regime) FCG. At increasing hydrogen pressure the FCG acceleration in Stage II almost does not change and shows a difference of approximately 30 times when compared to FCG in air. On the other hand, the transition point between the two regimes is strongly affected by the hydrogen pressure.

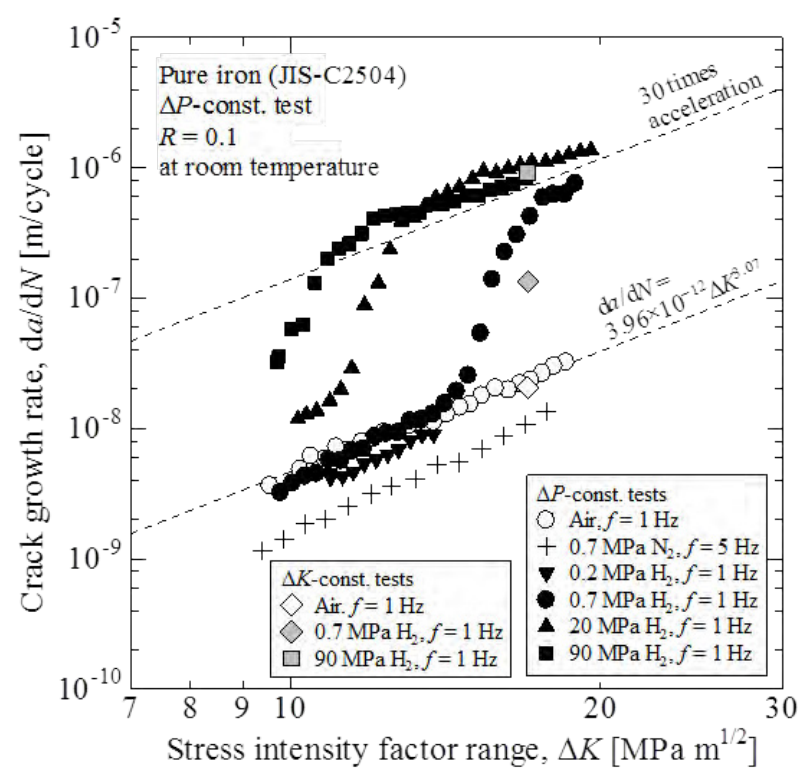

Fig. 1. FCG curves of pure Fe measured in laboratory air, 0.2 MPa nitrogen and 0.2, 0.7, 20, 90 MPa hydrogen gas.

Namely, increasing the hydrogen pressure made the transitional stress intensity factor range, $\Delta K^{\mathrm{T}}$, from Stage I to II to shift to lower values. The trends shown in Fig. 1 are common in other BCC materials [16].

\subsection{Fracture surface morphology}

Fracture surfaces of samples fatigued in air, 0.7 MPa, $20 \mathrm{MPa}$, and $90 \mathrm{MPa}$ hydrogen at different $\Delta K$ values are represented in Fig. 2. Fracture surfaces of specimens fatigued in air were covered only by ductile features (striation-like stripe patterns) throughout the tested $\Delta K$ range and fracture was fully transgranular (TG). On the other hand, the fracture surfaces of the specimens fatigued in hydrogen can be distinguished into two different regions: ductile transgranular fracture with a varying fraction of IG fracture was observed in regions corresponding to Stage I (Fig. 2c, e) and QC features 

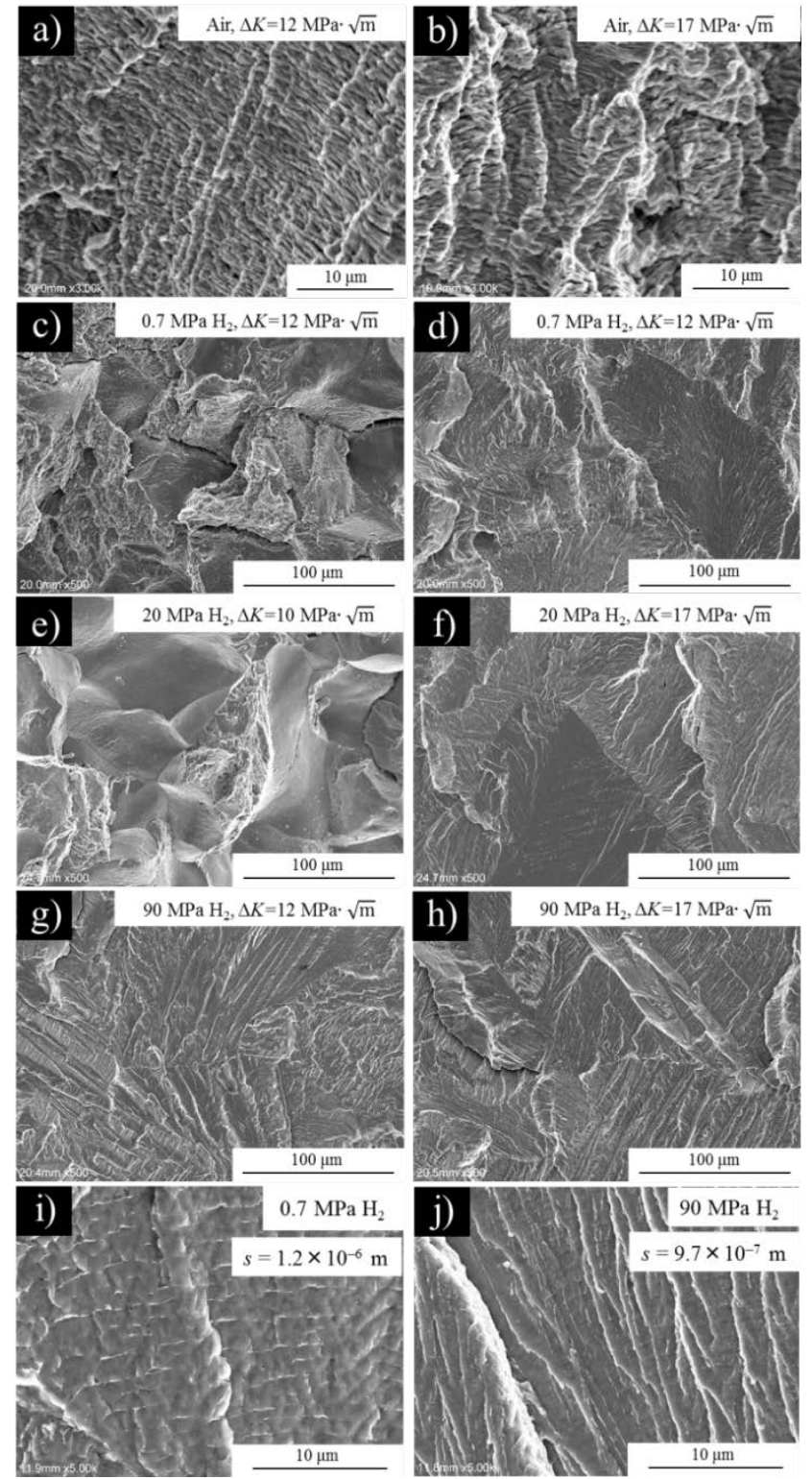

Fig. 2. SEM images of the fracture surfaces of the specimens subjected to $\Delta P$-constant tests in $(\mathrm{a}, \mathrm{b})$ air, (c, d) $0.7 \mathrm{MPa}$ hydrogen, and (e, f) $20 \mathrm{MPa}$ hydrogen, (g, h) $90 \mathrm{MPa}$ hydrogen at different $\Delta K$ values. (c, e) and $(\mathrm{d}, \mathrm{f}, \mathrm{g}, \mathrm{h})$ correspond to stage I and II crack growth regime respectively. High magnification images show striation spacing in (i) $0.7 \mathrm{MPa}$ hydrogen and (j) $90 \mathrm{MPa}$ hydrogen gas.

decorated by brittle-like striations dominated the surfaces corresponding to Stage II (Fig. 2d, f, g, h) FCG. In addition, it is confirmed that the spacing between brittle-like striations on QC fracture surfaces (Fig. 2i, j) match with the macroscopic FCG rate, which indicates that these striations form on a cycle-by-cycle basis. Fraction of intergranular fracture in Stage I increased at increasing hydrogen pressures. A similar transition from IG to QC fracturing between Stages I and II has also been reported in some pipeline steels $[17,18]$ tested in hydrogen gas.

A summarized overview of fracture feature distribution across the tested $\Delta K$ ranges is represented in Fig. 3. The onset of the appearance of QC features on the fracture surface coincides very well with the transition point of FCGR from Stage I to Stage II regime - the $\Delta K^{\mathrm{T}}$ value. In addition, the fraction of QC features reaches close to $100 \%$ surface coverage at the point where the transition ends and FCG curve becomes parallel to the one in air, but at higher level. Therefore, FCG acceleration enhancement was controlled by the coverage of QC fracture, and as it reaches $100 \%$ there is no further enhancement by increasing hydrogen pressures. On the other hand, higher hydrogen pressures caused a shift of $\Delta K^{\mathrm{T}}$ to the lower values implying that a combination of critical stress and hydrogen concentration is responsible for triggering QC type fracture.

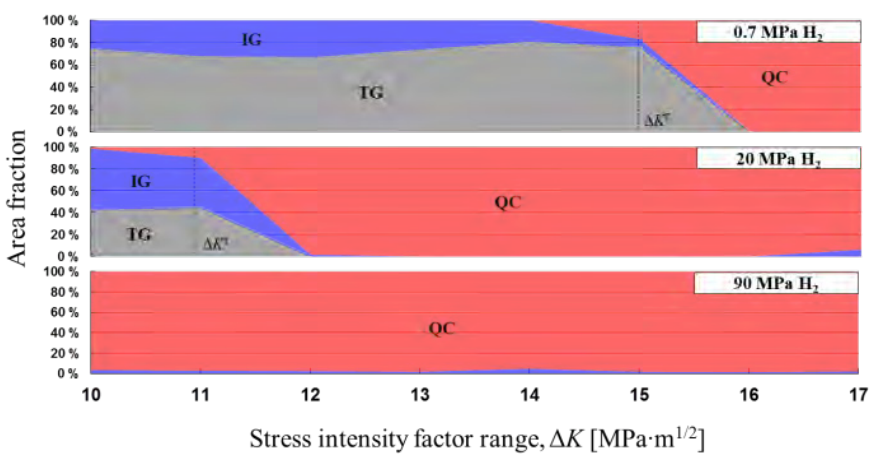

Fig. 3. Statistical overview of the fraction of fracture surface features at different $\Delta K$ values.

\subsection{Plastic deformation and dislocation structure analyses by EBSD and ECC imaging}

In order to reveal the path of the crack growth we acquired EBSD maps at the crack wake area and to visualize plastic deformation GROD analyses on the specimens fatigued at $\Delta K=17 \mathrm{MPa} \cdot \mathrm{m}^{1 / 2}$ (Fig. 4) were performed. As visible from the illustrations the crack path in air (Fig. 4a) is purely transgranular, wavy and branches in several locations. In comparison, the crack path in case of hydrogen (Fig. 4b, c) is also transgranural, but much more straight with path direction changing mostly across the grain boundaries. The plasticity analysis by using GROD maps on Stage II FCG (Fig. 4d, e, f) showed significant differences in samples fatigued in air and hydrogen gas. To evaluate these results in a more quantitative way we display a MADPDD graphs corresponding to the GROD maps in Fig 5. In case of air the sample was strongly plastically deformed up to $200 \mu \mathrm{m}$ away from the crack (Fig. 4d) and strain induced grain misorientation reached more than $40^{\circ}$ (Fig. 5). However, in case of $0.7 \mathrm{MPa}$ hydrogen the amount of plasticity was largely reduced and confined to the areas where the crack path was changing direction, while regions where the crack was running straight through the grains the plastic deformation was minimal. These observations are even more pronounced in the sample fatigued at $90 \mathrm{MPa}$ hydrogen. As visible from Fig. 5 the maximum misorientation angle is drastically reduced for higher hydrogen pressures indicating that plasticity is not just localized as suggested in previous studies $[9,19]$, but also reduced. Interestingly enough, the plastic deformation differences 


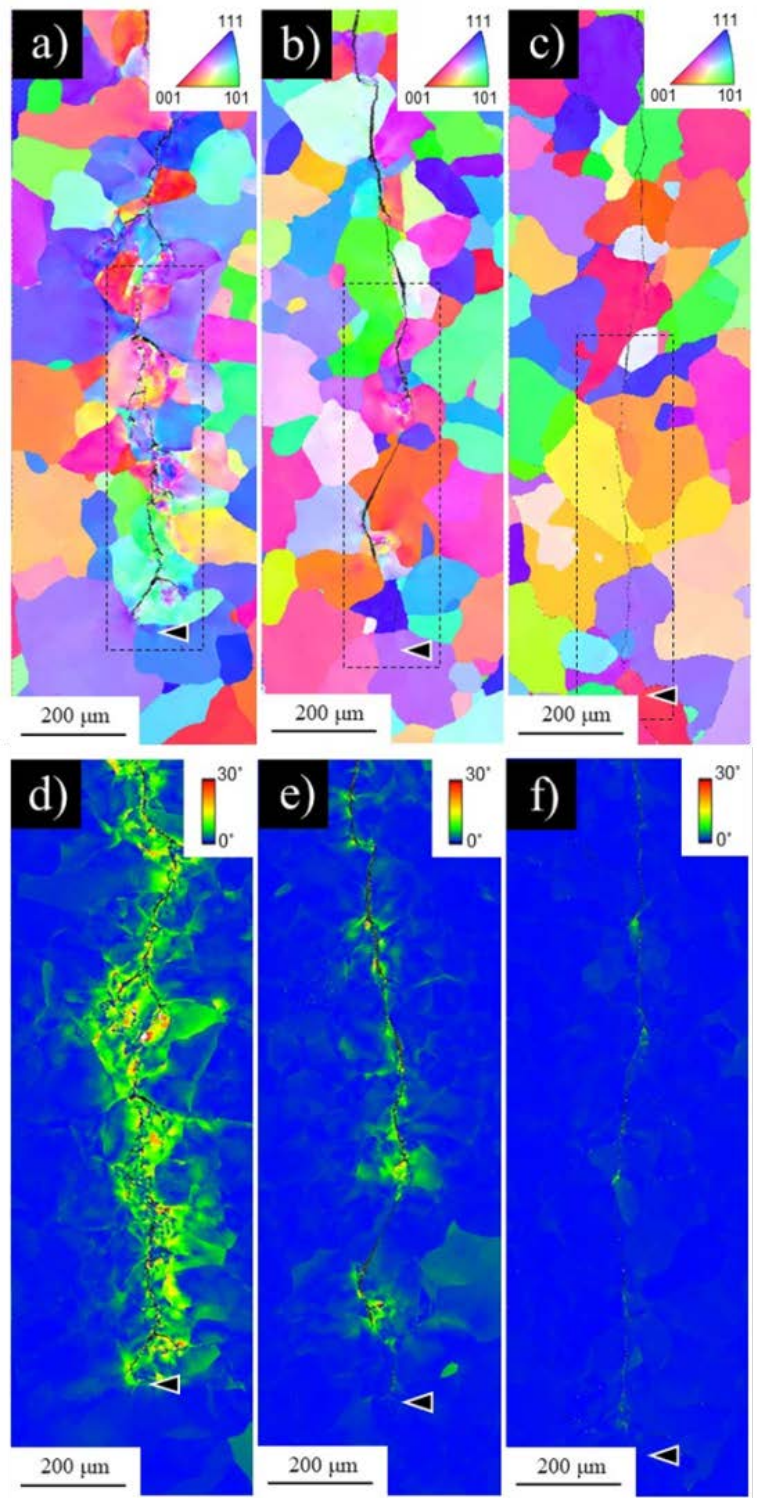

Fig. 4. SEM images of the fracture surfaces of the specimens subjected to $\Delta P$-constant tests in (a, b) air, (c, d) $0.7 \mathrm{MPa}$ hydrogen, and (e, f) $90 \mathrm{MPa}$ hydrogen at different $\Delta K$ values. $(c, e)$ and $(d, f)$ correspond to stage I and II crack growth regime respectively. The crack growth directions are bottom to top.

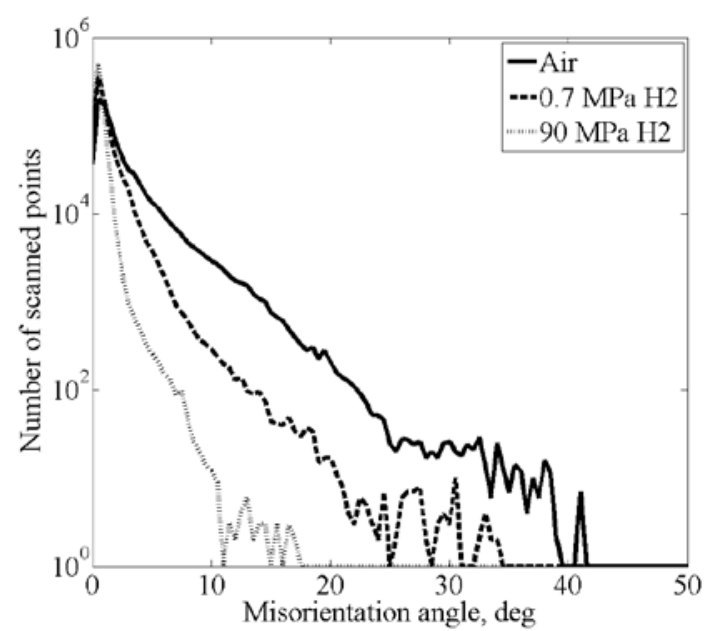

Fig. 5. MADPDD graph obtained from the GROD maps indicated in Fig. 4d, e, f. between $0.7 \mathrm{MPa}$ and $90 \mathrm{MPa}$ hydrogen do not have very large influence on FCG (Fig. 1) confirming our previous assertion that it is the type of fracture, which has major influence on FCG, while the amount of plasticity plays a secondary role.

The ECC images of the plastic deformation induced substructures formed in the regions marked by black dashed rectangles in Fig. 4a-c are shown in Fig. 6. In the sample fatigued in air a heavy plastic deformation resulted in a formation of fine sub-grains close to the crack and deformed regions further away from the crack (Fig. 6a). In contrast, the sample fatigued in $0.7 \mathrm{MPa}$ hydrogen gas demonstrated a much less distorted microstructure with sub-grain formation only in the most deformed regions (Fig. 6b). In case of $90 \mathrm{MPa}$ the microstructure is almost free of distortions and no subgrain formation was detected. $\{100\}$ type plane traces determined by EBSD analysis are indicated on two grains in Fig. 6b, c implying the crack growing along $\{100\}$ type cleavage plane. However, EBSD analysis provides only a 2 dimensional picture of the lateral surface, and it is not known how the crack plane is oriented deeper into the sample. Therefore, additional examination is needed to assure this assumption.

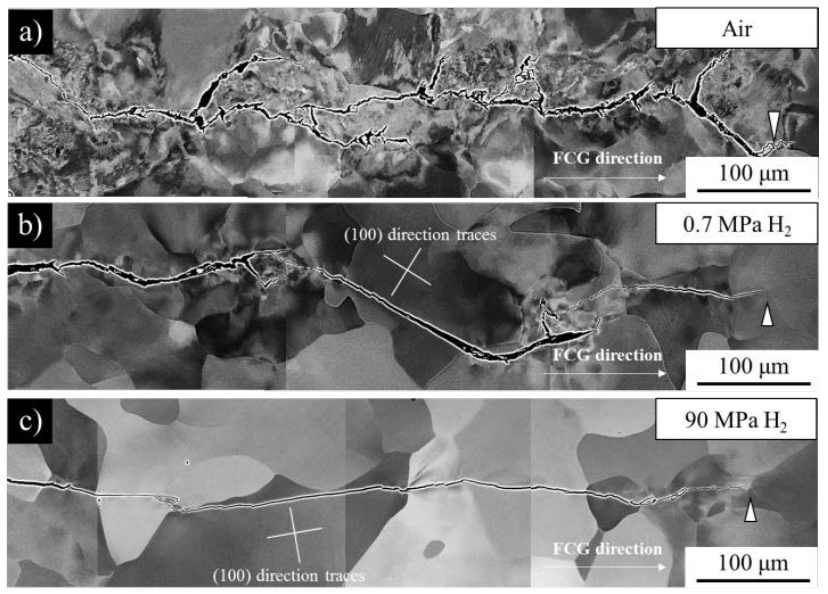

Fig. 6. ECC images of the crack wakes from samples fatigued in a) air, b) $0.7 \mathrm{MPa} \mathrm{H}_{2}$ and c) $90 \mathrm{MPa} \mathrm{H}_{2}$. The captured areas correspond to the regions marked by black dashed rectangles in Fig. 4a-c. The black arrowheads indicate crack tip positions, while yellow arrowheads show the fracture path along cleavage plane.

\subsection{Dislocation structures characterized by TEM}

Thin-foil samples for TEM were directly extracted from the ductile striations formed in air and QC regions formed in hydrogen gas using FIB, and the dislocation structure immediately beneath the fracture surfaces were examined by dark field-scanning TEM (DF-STEM) observation and are represented in Fig. 7. Dislocation cells were seen to dominate the dislocation structure in the sample fatigued in air (Fig. 8a) with a uniform cell size distribution. However, below the QC fracture surface formed in hydrogen gas (Fig. 8b, c), only dislocation tangles and individual dislocations were observed. According to the past studies [20-22], 
dislocation evolution is directly linked to the plastic strain amplitude applied to the material. At initial stages, individual dislocations and dislocation tangles are formed, and further plastic deformation forces dislocations to redistribute into veins, walls and finally cell structure. Applying these findings to our work, we can state that samples fatigued in air sustained high plastic strain amplitude, while samples fatigued in hydrogen suffered from much lower plastic strain amplitude. In addition, due to the fact that FIB lamellas were extracted from the regions, where fracture surface was perpendicular to the plane of lamella, we can recover the information about the fracture plane by using selected area diffraction in TEM. The diffraction patterns acquired in TEM indicate that in samples fatigued in hydrogen the QC fracture plane corresponds very well with the $\{100\}$ cleavage plane. These results strongly support the statement that hydrogen heavily suppresses plasticity evolution and promotes cleavage fracture when the hydrogen concentration and stress level ahead of the crack tip reaches critical values.

In a study on low-carbon steel by Nishikawa et al. [8], the proposed model for brittle striation formation during fatigue in hydrogen consists of three subsequent processes: i) crack tip opening; ii) microscopic defect nucleation ahead of the crack tip due to hydrogenenhanced stabilization of point defects [14,23], iii) final crack growth by a coalescence of the main crack with the primary defects due to ductile tearing. A crucial detail in this model is that crack growth progresses by ductile tearing, meaning that crack wake should contain significant plastic deformation and fracture surface should be dimpled on microscopic scale. However, they did not indicate the direct evidence of these dimple features ahead of the crack tip, and our findings of underdeveloped dislocation structures and relatively flat fracture surface between the striations contradict this model. Additionally, the preferential fracture path along $\{100\}$ cleavage plane observed in our study cannot be explained according to the ductile tearing process.

According to Troiano and Oriani [10,11], when hydrogen atoms enter the material they reduce the cohesive force between matrix atoms and activate the fracture under lower stress level than when hydrogen is absent. This model fits very well with our observations. Another interesting idea was introduced recently by Hajilou et al. [24]. The authors found similar plasticity suppression during the bending test using micro-sized cantilever of $\mathrm{Fe}-3 \% \mathrm{Si}$ alloy under in-situ electrochemical hydrogen-charging. According to the authors, large amount of hydrogen is attracted to the crack tip hydrostatic stress field and locks dislocations from motion, thereby leading to brittle type fracture. On a microscopic scale, a direct molecular dynamics calculation conducted by Song and Curtin also supported cleavage cracking in pure iron, due to the inhibited dislocation emission from crack tip source caused by hydrogen aggregation [25]. To some extent, all of these hypotheses can be applied to the present case to describe cleavage-like fracture. However, it is important to note that the dislocation density in the specimens fatigued in hydrogen gas is not negligible, and the cleavage fracture may be supported by the presence of these dislocations for example by assisting transport [26,27] or trapping $[28,29]$ of hydrogen.

As a result, we propose the following sequence for fatigue crack growth in hydrogen atmosphere during one loading cycle: i) as the cycle starts, the crack tip is being blunted by alternating slip mechanism and dislocations are being emitted from the crack tip, which may result in the final dislocation tangle structure visible in postmortem samples (Fig. 7b, c); ii) a maximum hydrostatic stress field is established just ahead of the main crack tip [30], which lowers the chemical potential and raises hydrogen concentration in this region; iii) the combination of critical stress and critical hydrogen concentration causes a primary cleavage micro crack formation ahead of the crack tip; iv) the main crack and the primary cleavage micro crack coalesce and propel the main crack forward until it is arrested. Striation spacing match with macroscopic FCGR indicates that the brittle striations on QC are formed on a cycle-bycycle basis, as was also confirmed in some conventional BCC steels [8,31]. This fact demonstrates that the macroscopic behavior of HAFCG in BCC iron is still controlled by a stable manner, even though on microscopic scale the fracture event is catastrophic.

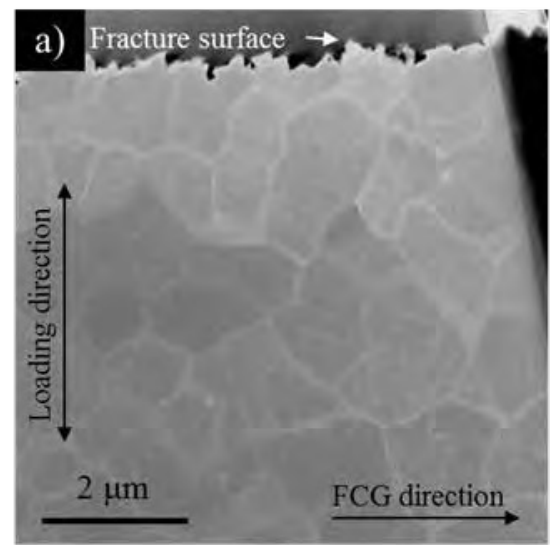

Air

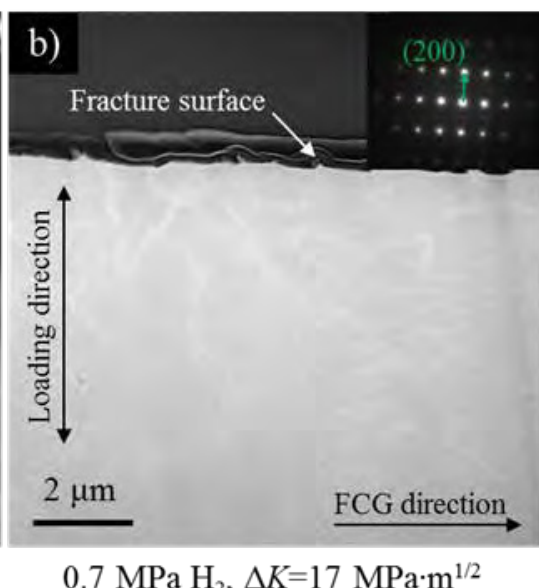

$0.7 \mathrm{MPa} \mathrm{H}_{2}, \Delta K=17 \mathrm{MPa} \cdot \mathrm{m}^{1 / 2}$

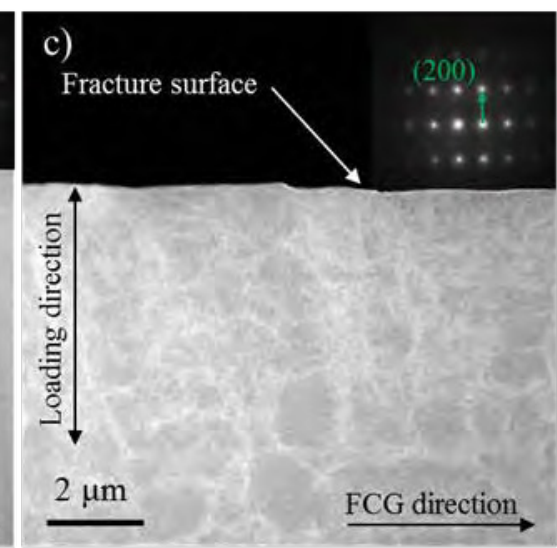

$90 \mathrm{MPa} \mathrm{H}_{2}, \Delta K=17 \mathrm{MPa} \cdot \mathrm{m}^{1 / 2}$

Fig. 7. Dislocation structures immediatly beneath the fracture surfaces of samples fatigued in a) air, b) $0.7 \mathrm{MPa} \mathrm{H}_{2}$, and c) 90 $\mathrm{MPa} \mathrm{H}_{2}$. The SADPs acquired from [110] zone axis are indicated in the insets. 


\section{Conclusions}

1. Hydrogen gas enhanced FCG acceleration in pure iron above critical stress intensity factor range, $\Delta K^{\mathrm{T}}$, by $\sim 30$ times and this enhancement was independent of hydrogen pressure. The $\Delta K^{\mathrm{T}}$ value was shifted to lower values by increasing hydrogen pressure.

2. The FCG acceleration beyond $\Delta K^{\mathrm{T}}$ manifested by the formation of hydrogen induced quasi-cleavage (QC) fracture and below $\Delta K^{\mathrm{T}}$ by intergranular (IG) fracture in combination with ductile transgranural features.

3. According to EBSD and ECCI analyses the plastic deformation and dislocation structure evolution around the crack wake were considerably reduced in case of hydrogen when the fracture surfaces are characterized by QC type features.

4. TEM micrographs showed dislocation tangle structure in hydrogen gas and dislocation cell structure in air, which were attributed to deformation at low plastic strain and high plastic strain amplitudes respectively. In addition, electron diffraction revealed the fact that the fracture in hydrogen was along $\{100\}$ crystal planes, implying that hydrogeninduced cleavage cracking was the main contributor to the HAFCG.

This work was supported by JSPS KAKENHI Grant Number JP16H04238 and JP16J02960. The Research Council of Norway is acknowledged for its support through the Norwegian Center for Transmission Electron Microscopy, NORTEM (197405/F50). This study also forms part of the "HIPP" project from the PETROMAKS2 program, funded by the Research Council of Norway [Grant Number: 102006899].

\section{References}

[1] M. Dadfarnia, A. Nagao, S. Wang, M. L. Martin, B. P. Somerday, and P. Sofronis, Int J Fract 196, 223 (2015).

[2] S. Suresh and R. O. Ritchie, Met. Sci. 16, 529 (1982).

[3] C. San Marchi and B. P. Somerday, Technical Reference for Hydrogen Compatibility of Materials (Albuquerque, 2012).

[4] R. P. Wei and D. Landes, Mater. Res. Stand. 12, 45 (1972).

[5] J. Yamabe, T. Matsumoto, S. Matsuoka, and Y. Murakami, Int. J. Fract. 177, 141 (2012).

[6] T. J. Marrow, P. J. Cotterill, and J. E. King, Acta Metall. Mater. 40, 2059 (1992).

[7] Y. Ogawa, D. Birenis, H. Matsunaga, A. Thøgersen, Ø. Prytz, O. Takakuwa, and J. Yamabe, Scr. Mater. 140, 13 (2017).

[8] H. Nishikawa, Y. Oda, and H. Noguchi, J. Solid Mech. Mater. Eng. 5, 370 (2011).

[9] S. Matsuoka, H. Tanaka, N. Homma, and Y. Murakami, Int. J. Fract. 168, 101 (2011).

[10] R. A. Oriani, Annu. Rev. Mater. Sci. 8, 327 (1978).

[11] A. R. Troiano, Trans. ASME 52, 54 (1960).

[12] C. D. Beachem, Metall. Trans. 3, 441 (1972).

[13] H. K. Birnbaum, Mechanisms of Hydrogen
Related Fracture of Metals (1989).

[14] M. Nagumo, ISIJ Int. 41, 590 (2001).

[15] Y. Ogawa, D. Birenis, H. Matsunaga, O. Takakuwa, J. Yamabe, Ø. Prytz, and A. Thøgersen, in 12th Int. Fatigue Congr. (2018).

[16] J. Yamabe, M. Yoshikawa, H. Matsunaga, and S. Matsuoka, Procedia Struct. Integr. 2, 525 (2016).

[17] B. P. Somerday, P. Sofronis, K. A. Nibur, C. San Marchi, and R. Kirchheim, Acta Mater. 61, 6153 (2013).

[18] R. L. Amaro, N. Rustagi, K. O. Findley, E. S. Drexler, and A. J. Slifka, Int. J. Fatigue 59, 262 (2014).

[19] Y. Murakami, T. Kanezaki, Y. Mine, and S. Matsuoka, Metall. Mater. Trans. A Phys. Metall. Mater. Sci. 39 A, 1327 (2008).

[20] H. Mughrabi, K. Herz, and X. Stark, Int. J. Fract. 17, 193 (1981).

[21] M. S. Pham and S. R. Holdsworth, Metall. Mater. Trans. A 45, 738 (2013).

[22] J. Polák, Fatigue of Steels (Elsevier B.V., 2007).

[23] K. Takai, H. Shoda, H. Suzuki, and M. Nagumo, Acta Mater. 56, 5158 (2008).

[24] T. Hajilou, Y. Deng, B. R. Rogne, N. Kheradmand, and A. Barnoush, Scr. Mater. 132, 17 (2017).

[25] J. Song and W. A. Curtin, Nat. Mater. 12, 145 (2012).

[26] J. Tien, A. W. Thompson, I. M. Bernstein, and R. J. Richards, Metall. Trans. A 7, 821 (1976).

[27] M. Dadfarnia, A. Nagao, S. Wang, M. L. Martin, B. P. Somerday, and P. Sofronis, Int. J. Fract. 196, 223 (2015).

[28] R. A. Oriani, Acta Metall. 18, 147 (1970).

[29] J. P. Hirth, Metall. Trans. A 11, 861 (1980).

[30] M.-J. Lii, X.-F. Chen, Y. Katz, and W. W. Gerberich, Acta Metall. Mater. 38, 2435 (1990).

[31] M. Yoshikawa, T. Matsuo, N. Tsutsumi, H. Matsunaga, and S. Matsuoka, Trans. JSME (in Japanese) 80, SMM0254 (2014). 